\title{
A Semilocal Convergence for a Uniparametric Family of Efficient Secant-Like Methods
}

\author{
I. K. Argyros, ${ }^{1}$ D. González, ${ }^{2}$ and Á. A. Magreñán ${ }^{2}$ \\ ${ }^{1}$ Department of Mathematical Sciences, Cameron University, Lawton, OK 73505, USA \\ ${ }^{2}$ Department of Mathematics and Computation, University of La Rioja, Logroño, 26006 La Rioja, Spain
}

Correspondence should be addressed to Á. A. Magreñán; alberto.magrenan@gmail.com

Received 8 May 2013; Accepted 21 October 2013; Published 6 February 2014

Academic Editor: Alfonso Montes-Rodriguez

Copyright (c) 2014 I. K. Argyros et al. This is an open access article distributed under the Creative Commons Attribution License, which permits unrestricted use, distribution, and reproduction in any medium, provided the original work is properly cited.

\begin{abstract}
We present a semilocal convergence analysis for a uniparametric family of efficient secant-like methods (including the secant and Kurchatov method as special cases) in a Banach space setting (Ezquerro et al., 2000-2012). Using our idea of recurrent functions and tighter majorizing sequences, we provide convergence results under the same or less computational cost than the ones of Ezquerro et al., (2013, 2010, and 2012) and Hernández et al., (2000, 2005, and 2002) and with the following advantages: weaker sufficient convergence conditions, tighter error estimates on the distances involved, and at least as precise information on the location of the solution. Numerical examples validating our theoretical results are also provided in this study.
\end{abstract}

\section{Introduction}

Let $U(x, r)$ and $\bar{U}(x, r)$ stand, respectively, for the open and closed ball in $\mathscr{X}$ with center $x \in \mathscr{X}$ and radius $r>0$. Denote by $\mathscr{L}(\mathscr{X}, \mathscr{Y})$ the space of bounded linear operators from $\mathscr{X}$ into $\mathcal{Y}$.

In this study, we are concerned with the problem of approximating a locally unique solution $x^{*}$ of nonlinear equation as follows:

$$
F(x)=0,
$$

where $F$ is a Fréchet-differentiable operator defined on a nonempty convex subset $\mathscr{D}$ of a Banach space $\mathscr{X}$ with values in a Banach space $\mathscr{Y}$.

Many problems from computational sciences, physics and other disciplines can be taken in the form of (1) using mathematical modelling [1-7]. The solution of these equations can rarely be found in closed form. That is why the solution methods for these equations are iterative. In particular, the practice of numerical analysis for finding such solutions is essentially connected to variants of Newton's method $[1,2,4-10]$. The study about the convergence of iterative procedures is usually focused on two types: semilocal and local convergence analysis. The semilocal convergence is, based on the information around an initial point, to give criteria ensuring the convergence of iterative procedure; while the local one is, based on the information around a solution, to find estimates of the radii of convergence balls. There are a lot of studies on the weakness and/or extension of the hypothesis made on the underlying operators; see, for example, [1-26] and the references therein.

Hernández and Rubio used in [22] the uniparametric family of secant-like methods defined by

$$
\begin{gathered}
x_{-1}, x_{0} \quad \text { given in } \mathscr{D}, \\
y_{n}=\mu x_{n}+(1-\mu) x_{n-1}, \quad \mu \in[0,1], \\
x_{n+1}=x_{n}-B_{n}^{-1} F\left(x_{n}\right), \quad B_{n}=\left[y_{n}, x_{n} ; F\right],
\end{gathered}
$$

for each $n=0,1, \ldots$,

and the method of recurrent relations to generate a sequence $\left\{x_{n}\right\}$ approximating $x^{*}$. Here, $[z, w ; F]$ for each $z, w \in \mathscr{D}$ is a divided difference of order one, which is a bounded linear operator such that $[1,4,6-8,14,15]$

$$
\begin{gathered}
{[z, w ; F]: \mathscr{D} \longrightarrow \mathscr{Y},} \\
{[z, w ; F](z-w)=F(z)-F(w) .}
\end{gathered}
$$


Secant-like method (2) can be considered as a combination of the secant and Newton's method. Indeed, if $\mu=0$, we obtain the secant method and if $\mu=1$, we get Newton's method provided that $F^{\prime}$ is Fréchet-differentiable on $\mathscr{D}$, since then $x_{n}=y_{n}$ and $\left[y_{n}, x_{n} ; F\right]=F^{\prime}\left(x_{n}\right)$.

It was shown in $[20,21]$ that the $R$-order of convergence is at least $(1+\sqrt{5}) / 2$ for $\lambda \in[0,1)$, the same as that of the secant method. Later in [5], another uniparametric family of secant-like methods defined by

$$
\begin{gathered}
x_{-1}, x_{0} \quad \text { given in } \mathscr{D}, \\
y_{n}=\lambda x_{n}+(1-\lambda) x_{n-1}, \quad \lambda \geq 1, \\
x_{n+1}=x_{n}-A_{n}^{-1} F\left(x_{n}\right), \quad A_{n}=\left[y_{n}, x_{n-1} ; F\right] \\
\text { for each } n=0,1, \ldots .
\end{gathered}
$$

was studied. It was shown that there exists $\lambda_{0} \geq 2$, and that the $R$-order of convergence is at least $(1+\sqrt{5}) / 2$ if $\lambda \in\left[1, \lambda_{0}\right]$ and $\lambda \neq 2$, and if $\lambda=2$, the $R$-order of convergence is quadratic. Note that if $\lambda=1$, we obtain the secant method, whereas if $\lambda=2$, we obtain the Kurchatov method $[4,5,7,8]$.

We present a semilocal convergence analysis for secantlike method (2) using our idea of recurrent functions instead of recurrent relations and tighter majorizing sequences. This way, our analysis provided the following advantages $(A)$ over the work in [5] under the same computational cost.

$\left(A_{1}\right)$ Weaker sufficient convergence conditions.

$\left(A_{2}\right)$ Tighter estimates on the distances $\left\|x_{n+1}-x_{n}\right\|$ and $\left\|x_{n}-x^{*}\right\|$ for each $n=0,1, \ldots$.

$\left(A_{3}\right)$ At least as precise information on the location of the solution.

$\left(A_{4}\right)$ The results are presented in affine invariant form, whereas the ones in [5] are given in nonaffine invariant forms. The advantages of affine versus nonaffine results have been explained in $[1,4,6-8,14,15]$.

Our hypotheses for the semilocal convergence of secantlike method (4) are as follows.

$\left(C_{1}\right)$ There exists a divided difference of order one $[z, w$; $F] \in \mathscr{L}(\mathscr{X}, \mathscr{Y})$ satisfying $(3)$.

$\left(C_{2}\right)$ There exist $x_{0} \in \mathscr{D}, \eta \geq 0$ such that $A_{0}^{-1} \in \mathscr{L}(\mathscr{Y}, \mathscr{X})$ and $\left\|A_{0}^{-1} F\left(x_{0}\right)\right\| \leq \eta$.

$\left(C_{3}\right)$ There exist $x_{-1}, x_{0} \in \mathscr{D}$ and $c \geq 0$ such that

$$
\left\|x_{0}-x_{-1}\right\| \leq c \text {. }
$$

$\left(C_{4}\right)$ There exists $K>0$ such that

$$
\begin{aligned}
\left\|A_{0}^{-1}([x, y ; F]-[v, w ; F])\right\| & \leq K(\|x-v\|+\|y-w\|) \\
& \text { for each } x, y, v, w \in \mathscr{D} .
\end{aligned}
$$

We will denote by $(C)$ conditions $\left(C_{1}\right)-\left(C_{4}\right)$. In view of $\left(C_{4}\right)$, there exist $H_{0}, H_{1}, H>0$ such that

$$
\left(C_{5}\right)\left\|A_{0}^{-1}\left(\left[x_{1}, x_{0} ; F\right]-A_{0}\right)\right\| \leq H_{0}\left(\left\|x_{1}-y_{0}\right\|+\left\|x_{0}-x_{-1}\right\|\right),
$$

$\left(C_{6}\right)\left\|A_{0}^{-1}\left(A_{1}-A_{0}\right)\right\| \leq H_{1}\left(\left\|y_{1}-y_{0}\right\|+\left\|x_{0}-x_{-1}\right\|\right)$,

$\left(C_{7}\right)\left\|A_{0}^{-1}\left([x, y ; F]-A_{0}\right)\right\| \leq H\left(\left\|x-y_{0}\right\|+\left\|y-x_{-1}\right\|\right)$ for each $x, y \in \mathscr{D}$.

Clearly,

$$
H_{0} \leq H_{1} \leq H \leq K
$$

hold in general and $\mathrm{K} / \mathrm{H}, \mathrm{H} / \mathrm{H}_{1}$ can be arbitrarily large $[1,2,4]$. Note that $\left(C_{5}\right),\left(C_{6}\right)$, and $\left(C_{7}\right)$ are not additional to $\left(C_{4}\right)$ hypotheses. In practise, the computation of $K$ requires the computation of $H_{0}, H_{1}$, and $H$. It also follows from $\left(C_{4}\right)$ that $F$ is differentiable $[1-3,8,9]$.

The paper is organized as follows. In Section 2, we show that under the same hypotheses as in [23] and using recurrent relations, we obtain at least as precise information on the location of the solution. Section 3 contains the semilocal convergence analysis using weaker hypotheses and recurrent functions. We also show the advantages $(A)$. The results are also extended to cover the case of equations with nondifferentiable operators. Numerical examples are presented in the concluding Section 4

\section{Semilocal Convergence Using Recurrent Relations}

As in [5], let us define sequences $\left\{a_{n}\right\}$ and $\left\{b_{n}\right\}$ for each $n=$ $0,1, \ldots$ by

$$
\begin{gathered}
a_{-1}=\frac{\eta}{c+\eta}, \quad b_{-1}=\frac{K c^{2}}{c+\eta}, \\
a_{n}=f\left(a_{n-1}\right) g\left(a_{n-1}\right) b_{n-1}, \\
b_{n}=f\left(a_{n-1}\right)^{2} a_{n-1} b_{n-1},
\end{gathered}
$$

and functions $f, g$ on $[0,1)$ by

$$
f(t)=\frac{1}{1-t}, \quad g(t)=(2-\lambda)+\lambda f(t) t
$$

Next, we present the main result in this section in affine invariant form.

Theorem 1. Under the $(C)$ hypotheses, further suppose that

$$
\bar{U}\left(x_{0}, R\right) \subseteq \mathscr{D},
$$

and for $\lambda \in\left[1, \lambda_{0}\right]$,

$$
a_{-1}<\frac{3-\sqrt{5}}{2}, \quad b_{-1}<\frac{a_{-1}\left(1-a_{-1}\right)^{2}}{2\left(1-a_{-1}\right)-\lambda\left(1-2 a_{-1}\right)},
$$

where

$$
R=\frac{1-a_{0}}{1-2 a_{0}} \lambda \eta, \quad \lambda_{0} \in\left[2, \frac{2 c}{c-\eta}\right)
$$

Then, sequence $\left\{x_{n}\right\}$ generated by secant-like method (4) is well defined, remains in $\bar{U}\left(x_{0}, R\right)$ for each $n=0,1,2, \ldots$, and 
converges to a solution $x^{*} \in \bar{U}\left(x_{0}, R\right)$ of equation $F(x)=0$. Moreover, the following estimates hold

$$
\begin{gathered}
\left\|x_{n+1}-x_{n}\right\| \leq f\left(a_{n-1}\right) a_{n-1}\left\|x_{n}-x_{n-1}\right\|, \\
\left\|x_{n}-x^{*}\right\| \leq \frac{\left(f\left(a_{0}\right) a_{0}\right)^{n}}{1-f\left(a_{0}\right) a_{0}}\left\|x_{1}-x_{0}\right\| .
\end{gathered}
$$

Furthermore, the solution $x^{*}$ is unique in $\mathscr{D}_{0}=U\left(x_{0}, \sigma_{0}\right) \cap D$, where $\sigma_{0}=(1 / H)-\lambda c-R$, provided that

$$
R<\frac{1}{2}\left(\frac{1}{H}-\lambda c\right)=R_{0} .
$$

Proof. The proof with the exception of the uniqueness part is given in Theorem 3 in [5] if we use $A_{0}^{-1} F$ instead of $F$ and set $b=1$, where $\left\|A_{0}^{-1}\right\| \leq b$.

To prove the uniqueness of the solution, let us assume that $y^{*} \in \mathscr{D}_{0}$ is a solution of $F(x)=0$. Let $L=\left[y^{*}, x^{*} ; F\right]$. Then, using $\left(C_{7}\right)$ and the definition of $\sigma_{0}$, we get in turn that

$$
\begin{aligned}
\left\|A_{0}^{-1}\left(L-A_{0}\right)\right\| & \leq H\left(\left\|y^{*}-y_{0}\right\|+\left\|x^{*}-x_{-1}\right\|\right) \\
& <H\left(\sigma_{0}+\lambda c+R\right)=1 .
\end{aligned}
$$

It follows from (15) and the Banach lemma on invertible operators $[1,2,4,6-8,14]$ that $L^{-1} \in \mathscr{L}(\mathscr{Y}, \mathscr{X})$. Using the identity $0=F\left(y^{*}\right)-F\left(x^{*}\right)=L\left(y^{*}-x^{*}\right)$, we deduce that $x^{*}=y^{*}$. That completes the proof of the theorem.

Remark 2. If $K=H$, Theorem 1 reduces to Theorem 3 in [5]. Otherwise, that is, if $H<K$, then our Theorem 1 constitutes an improvement over Theorem 3 , since

$$
\begin{gathered}
\sigma<\sigma_{0}, \\
R_{0}<R_{1},
\end{gathered}
$$

where

$$
\sigma=\frac{1}{K}-\lambda c-R, \quad R_{0}=\frac{1}{2}\left(\frac{1}{K}-\lambda c\right),
$$

where given in [5] (for $b=1$ ). Hence, (16) justify our claim for this section which was made in the Introduction of this study.

\section{Semilocal Convergence Using Recurrent Functions}

We present the semilocal convergence of secant-like methods. First, we need some auxiliary results on majorizing sequences for secant-like method.

Lemma 3. Let $c \geq 0, \eta>0, H>0, K>0$ and $\lambda \geq 1$. Set $t_{-1}=0, t_{0}=c$, and $t_{1}=c+\eta$. Define scalar sequences $\left\{q_{n}\right\}$, $\left\{t_{n}\right\},\left\{\alpha_{n}\right\}$ for each $n=0,1, \ldots$ by

$$
\begin{gathered}
q_{n}=H \lambda\left(t_{n+1}+t_{n}-c\right), \\
t_{n+2}=t_{n+1}+\frac{K\left(t_{n+1}-t_{n}+\lambda\left(t_{n}-t_{n-1}\right)\right)}{1-q_{n}}\left(t_{n+1}-t_{n}\right), \\
\alpha_{n}=\frac{K\left(t_{n+1}-t_{n}+\lambda\left(t_{n}-t_{n-1}\right)\right)}{1-q_{n}},
\end{gathered}
$$

functions $f_{n}$ on $[0,1)$ for each $n=1,2, \ldots$ by

$$
\begin{aligned}
f_{n}(t)= & K\left(t^{n}+\lambda t^{n-1}\right) \eta \\
& +H \lambda\left(\left(1+t+\cdots+t^{n+1}\right) \eta\right. \\
& \left.+\left(1+t+\cdots+t^{n}\right) \eta+c\right)-1,
\end{aligned}
$$

and polynomial $p$ on $[0,1)$ by

$$
p(t)=H \lambda t^{3}+(H \lambda+K) t^{2}+K(\lambda-1) t-\lambda K .
$$

Denote by $\alpha$ the only root of polynomial $p$ in $(0,1)$. Suppose that

$$
0 \leq \alpha_{0} \leq \alpha \leq \frac{1-H \lambda(c+2 \eta)}{1-H \lambda c}
$$

Then, sequence $\left\{t_{n}\right\}$ is nondecreasing, bounded from above by $t^{* *}$ that is defined by

$$
t^{* *}=\frac{\eta}{1-\alpha}+c
$$

and converges to its unique least upper bound $t^{*}$ which satisfies

$$
c+\eta \leq t^{*} \leq t^{* *} \text {. }
$$

Moreover, the following estimates are satisfied for each $n=$ $0,1,2, \ldots$ :

$$
\begin{gathered}
0 \leq t_{n+1}-t_{n} \leq \alpha^{n} \eta, \\
t^{*}-t_{n} \leq \frac{\alpha^{n} \eta}{1-\alpha} .
\end{gathered}
$$

Proof. We will first show that polynomial $p$ has roots in $(0,1)$. Indeed, we have $p(0)=-\lambda K<0$ and $p(1)=2 H \lambda>0$. Using the intermediate value theorem, we deduce that there exists at least one root of $p$ in $(0,1)$. Moreover $p^{\prime}(t)>0$. Hence, $p$ crosses the positive axis only once. Denote by $\alpha$ the only root of $p$ in $(0,1)$. It follows from (18) and (19) that estimate (25) is certainly satisfied if

$$
0 \leq \alpha_{n} \leq \alpha .
$$

Estimate (27) is true by (22) for $n=0$. Then, we have by (18) that

$$
\begin{aligned}
t_{2}-t_{1} & \leq \alpha\left(t_{1}-t_{0}\right) \Longrightarrow t_{2} \leq t_{1}+\alpha\left(t_{1}-t_{0}\right) \\
& \Longrightarrow t_{2} \leq \eta+t_{0}+\alpha \eta \\
& =c+(1+\alpha) \eta=c+\frac{1-\alpha^{2}}{1-\alpha} \eta<t^{* *} .
\end{aligned}
$$

Suppose that

$$
t_{k+1}-t_{k} \leq \alpha^{k} \eta, \quad t_{k+1} \leq c+\frac{1-\alpha^{k+1}}{1-\alpha} \eta
$$

for each $k \leq n$. 
Estimate (27) will be true for $k+1$ replacing $n$ if

$$
0 \leq \alpha_{k+1} \leq \alpha
$$

or

$$
f_{k}(\alpha) \leq 0
$$

where $f_{k}$ is defined by (20). We need a relationship between two consecutive recurrent functions $f_{k}$ for each $k=1,2 \ldots$.. Using (20) and (21), we deduce that

$$
f_{k+1}(\alpha)=f_{k}(\alpha)+p(\alpha) \alpha^{k-1} \eta=f_{k}(\alpha),
$$

since $p(\alpha)=0$. Define function $f_{\infty}$ on $(0,1)$ by

$$
f_{\infty}(t)=\lim _{k \rightarrow+\infty} f_{k}(t)
$$

Then, we get from (20) and (33) that

$$
f_{\infty}(\alpha)=H \lambda\left(\frac{2 \eta}{1-\alpha}+c\right)-1
$$

Hence, by (32)-(34), (31) is satisfied if

$$
f_{\infty}(\alpha) \leq 0
$$

which is true by (22). The induction for (25) is complete. That is, sequence $\left\{t_{n}\right\}$ is nondecreasing, bounded from above by $t^{* *}$ that is given by (23), and as such it converges to some $t^{*}$ which satisfies (24). Estimate (26) follows from (25) by using standard majorization techniques $[1,2,4,6-8,14]$. The proof of Lemma 3 is complete.

Lemma 4. Let $c \geq 0, \eta>0, H_{0}>0, H_{1}>0, H>0, K>0$, and $\lambda \geq 1$. Set $s_{-1}=0, s_{0}=c$, and $s_{1}=c+\eta$. Define scalar sequences $\left\{s_{n}\right\},\left\{b_{n}\right\}$ for each $n=1,2, \ldots$ by

$$
\begin{gathered}
s_{2}=s_{1}+\frac{H_{0}\left(s_{1}-s_{0}+\lambda\left(s_{0}-s_{-1}\right)\right)}{1-H_{1} \lambda\left(s_{1}+s_{0}-c\right)}\left(s_{1}-s_{0}\right), \\
s_{n+2}=s_{n+1}+\frac{K\left(s_{n+1}-s_{n}+\lambda\left(s_{n}-s_{n-1}\right)\right)}{1-H \lambda\left(s_{n+1}+s_{n}-c\right)}\left(s_{n+1}-s_{n}\right), \\
b_{1}=\frac{H_{0}\left(s_{1}-s_{0}+\lambda\left(s_{0}-s_{-1}\right)\right)}{1-H_{1} \lambda\left(s_{1}+s_{0}-c\right)}, \\
b_{n}=\frac{K\left(s_{n+1}-s_{n}+\lambda\left(s_{n}-s_{n-1}\right)\right)}{1-H \lambda\left(s_{n+1}+s_{n}-c\right)},
\end{gathered}
$$

and functions $g_{n}$ on $[0,1)$ by

$$
\begin{aligned}
g_{n}(t)= & K(t+\lambda) t^{n-1}\left(s_{2}-s_{1}\right) \\
& +H \lambda t\left(2 s_{1}+\frac{1-t^{n+1}}{1-t}\left(s_{2}-s_{1}\right)+\frac{1-t^{n}}{1-t}\left(s_{2}-s_{1}\right)\right) \\
& -(1+H \lambda c) t .
\end{aligned}
$$

Suppose that

$$
0 \leq b_{1} \leq \alpha \leq \frac{1-H \lambda\left(2 s_{2}-c\right)}{1-H \lambda\left(2 s_{1}-c\right)},
$$

where $\alpha$ is defined in Lemma 3. Then, sequence $\left\{s_{n}\right\}$ is nondecreasing, bounded from above by $s^{* *}$ that is defined by

$$
s^{* *}=c+\eta+\frac{s_{2}-s_{1}}{1-\alpha},
$$

and converges to its unique least upper bound ${ }^{*}$ which satisfies

$$
c+\eta \leq s^{*} \leq s^{* *} \text {. }
$$

Moreover, the following estimates are satisfied for each $n=$ $1,2, \ldots$ :

$$
0 \leq s_{n+2}-s_{n+1} \leq \alpha^{n}\left(s_{2}-s_{1}\right) .
$$

Proof. We will show using induction that

$$
0 \leq b_{n} \leq \alpha .
$$

Estimate (43) is true for $n=0$ by (39). Then, we have by (36) that

$$
\begin{aligned}
0 & \leq s_{3}-s_{2} \leq \alpha\left(s_{2}-s_{1}\right) \\
& \Longrightarrow s_{3} \leq s_{2}+\alpha\left(s_{2}-s_{1}\right) \\
& \Longrightarrow s_{3} \leq s_{2}+(1+\alpha)\left(s_{2}-s_{1}\right) \\
- & \left(s_{2}-s_{1}\right) \Longrightarrow s_{3} \leq s_{1}+\frac{1-\alpha^{2}}{1-\alpha}\left(s_{2}-s_{1}\right) \leq s^{* *}
\end{aligned}
$$

Suppose that (43) holds for each $n \leq k$. Then, using (36), we get that

$$
\begin{aligned}
& 0 \leq s_{k+2}-s_{k+1} \leq \alpha^{k}\left(s_{2}-s_{1}\right), \\
& s_{k+2} \leq s_{1}+\frac{1+\alpha^{k+1}}{1-\alpha}\left(s_{2}-s_{1}\right) .
\end{aligned}
$$

Estimate (43) will be satisfied if

$$
g_{k}(\alpha) \leq 0 .
$$

Using (38), we get the following relationship between two consecutive recurrent functions $g_{k}$ :

$$
g_{k+1}(\alpha)=g_{k}(\alpha)+p(\alpha) \alpha^{k-1}\left(s_{2}-s_{1}\right)=g_{k}(\alpha) .
$$

Define function $g_{\infty}$ on $[0,1)$ by

$$
g_{\infty}(t)=\lim _{k \rightarrow+\infty} g_{k}(t)
$$

Then, we get from (38) that

$$
g_{\infty}(\alpha)=2 \alpha H \lambda\left[s_{1}+\frac{s_{2}-s_{1}}{1-\alpha}\right]-\alpha(1+H \lambda c) .
$$

Then, (46) is satisfied if

$$
g_{\infty}(\alpha) \leq 0,
$$

which is true by the choice of $\alpha$ and the right hand side inequality in hypothesis (39). The induction for (43) (i.e., (42)) is complete. The rest of the proof as identical to Lemma 3 is omitted. The proof is complete. 
Remark 5. (a) Let us consider an interesting choice for $\lambda$. Let $\lambda=1$ (secant method). Then, using (21) and (22), we have that

$$
\begin{gathered}
\alpha=\frac{2 K}{K+\sqrt{K^{2}+4 H K}} \\
\frac{K(c+\eta)}{1-H(c+\eta)} \leq \alpha \leq \frac{1-H(c+2 \eta)}{1-H c} .
\end{gathered}
$$

The corresponding condition for the secant method is given by $[2,4,9,23]$ as follows:

$$
K c+2 \sqrt{K \eta} \leq 1
$$

Condition (52) can be weaker than (53) (see also the numerical examples at the end of the study). Moreover, the majorizing sequence $\left\{u_{n}\right\}$ for the secant method related to (53) is given by

$$
\begin{gathered}
u_{-1}=0, \quad u_{0}=c, \quad u_{1}=c+\eta, \\
u_{n+2}=u_{n+1}+\frac{K\left(u_{n+1}-u_{n-1}\right)}{1-K\left(u_{n+1}+u_{n}-c\right)}\left(u_{n+1}-u_{n}\right) .
\end{gathered}
$$

A simple inductive argument shows that if $H<K$, then for each $n=2,3, \ldots$ :

$$
\begin{gathered}
t_{n}<u_{n}, \quad t_{n+1}-t_{n}<u_{n+1}-u_{n}, \\
t^{*} \leq u^{*}=\lim _{n \rightarrow+\infty} u_{n} .
\end{gathered}
$$

(b) The majorizing sequence $\left\{v_{n}\right\}$ used in [5] is essentially given by

$$
\begin{gathered}
v_{-1}=0, \quad v_{0}=c, \quad v_{1}=c+\eta, \\
v_{n+2}=v_{n+1}+\frac{K\left(v_{n+1}-v_{n}+\lambda\left(v_{n}-v_{n-1}\right)\right)}{1-K \lambda\left(v_{n+1}+v_{n}-c\right)}\left(v_{n+1}-v_{n}\right) .
\end{gathered}
$$

Then, again we have

$$
\begin{gathered}
t_{n}<v_{n}, \quad t_{n+1}-t_{n}<v_{n+1}-v_{n}, \\
t^{*} \leq v^{*}=\lim _{n \rightarrow+\infty} v_{n} .
\end{gathered}
$$

Moreover, our sufficient convergence conditions can be weaker than [5].

(c) Clearly, iteration $\left\{s_{n}\right\}$ is tighter than $\left\{t_{n}\right\}$ and as we have in (57) than for $H_{0}<K$ or $H_{1}<H$ as follows:

$$
\begin{gathered}
s_{n}<t_{n}, \quad s_{n+1}-s_{n}<t_{n+1}-t_{n}, \\
s^{*}=\lim _{n \rightarrow+\infty} s_{n}<t^{*} .
\end{gathered}
$$

Next, we present obvious and useful extensions of Lemmas 3 and 4 , respectively.
Lemma 6. Let $N=0,1,2, \ldots$ be fixed. Suppose that

$$
\begin{gathered}
t_{1} \leq t_{2} \leq \cdots \leq t_{N} \leq t_{N+1}, \\
\frac{1}{H \lambda}>t_{N+1}-t_{N}+\lambda\left(t_{N}-t_{N-1}\right), \\
0 \leq \alpha_{N} \leq \alpha \leq \frac{1-H \lambda\left(t_{N}-t_{N-1}+2\left(t_{N+1}-t_{N}\right)\right)}{1-H \lambda\left(t_{N}-t_{N-1}\right)} .
\end{gathered}
$$

Then, sequence $\left\{t_{n}\right\}$ generated by (19) is nondecreasing, bounded from above by $t^{* *}$, and converges to $t^{*}$ which satisfies $t^{*} \epsilon$ $\left[t_{N+1}, t^{*}\right]$. Moreover, the following estimates are satisfied for each $n=0,1, \ldots$ :

$$
\begin{gathered}
0 \leq t_{N+n+1}-t_{N+n} \leq \alpha^{n}\left(t_{N+1}-t_{N}\right), \\
t^{*}-t_{N+n} \leq \frac{\alpha^{n}}{1-\alpha}\left(t_{N+1}-t_{N}\right) .
\end{gathered}
$$

Lemma 7. Let $N=1,2, \ldots$ be fixed. Suppose that

$$
\begin{gathered}
s_{1} \leq s_{2} \leq \cdots \leq s_{N} \leq s_{N+1}, \\
\frac{1}{H \lambda}>s_{N+1}-s_{N}+\lambda\left(s_{N}-s_{N-1}\right), \\
0 \leq b_{N} \leq \alpha \leq \frac{1-H \lambda\left(2 s_{N+1}-s_{N-1}\right)}{1-H \lambda\left(2 s_{N}-s_{N-1}\right)} .
\end{gathered}
$$

Then, sequence $\left\{s_{n}\right\}$ generated by (36) is nondecreasing, bounded from above by $s^{* *}$ and converges to $s^{*}$ which satisfies $s^{*} \epsilon$ $\left[s_{N+1}, s^{*}\right]$. Moreover, the following estimates are satisfied for each $n=0,1, \ldots$

$$
\begin{gathered}
0 \leq s_{N+n+1}-s_{N+n} \leq \alpha^{n}\left(s_{N+1}-s_{N}\right), \\
s^{*}-s_{N+n} \leq \frac{\alpha^{n}}{1-\alpha}\left(s_{N+1}-s_{N}\right) .
\end{gathered}
$$

Next, we present the following semilocal convergence result for secant-like method under the $(C)$ conditions.

Theorem 8. Suppose that the (C), Lemma 3 (or Lemma 6) conditions and

$$
U=\bar{U}\left(x_{0},(2 \lambda-1) t^{*}\right) \subseteq \mathscr{D}
$$

hold. Then, sequence $\left\{x_{n}\right\}$ generated by secant-like method is well defined, remains in $U$ for each $n=-1,0,1,2, \ldots$, and converges to a solution $x^{*} \in \bar{U}\left(x_{0}, t^{*}-c\right)$ of equation $F(x)=0$. Moreover, the following estimates are satisfied for each $n=$ $0,1, \ldots$ :

$$
\begin{gathered}
\left\|x_{n+1}-x_{n}\right\| \leq t_{n+1}-t_{n}, \\
\left\|x_{n}-x^{*}\right\| \leq t^{*}-t_{n} .
\end{gathered}
$$

Furthermore, if there exists $T \geq t^{*}-c$ such that

$$
\begin{gathered}
\bar{U}\left(x_{0}, r\right) \subseteq \mathscr{D}, \\
H\left(T+t^{*}+(\lambda-1) c\right)<1,
\end{gathered}
$$

then, the solution $x^{*}$ is unique in $\bar{U}\left(x_{0}, T\right)$. 
Proof. We use mathematical induction to prove that

$$
\begin{aligned}
\left\|x_{k+1}-x_{k}\right\| & \leq t_{k+1}-t_{k}, \\
\bar{U}\left(x_{k+1}, t^{*}-t_{k+1}\right) & \subseteq \bar{U}\left(x_{k}, t^{*}-t_{k}\right),
\end{aligned}
$$

for each $k=-1,0,1, \ldots$ Let $z \in \bar{U}\left(x_{0}, t^{*}-t_{0}\right)$. Then, we obtain that

$$
\begin{aligned}
\left\|z-x_{-1}\right\| & \leq\left\|z-x_{0}\right\|+\left\|x_{0}-x_{-1}\right\| \leq t^{*}-t_{0}+c=t^{*} \\
& =t^{*}-t_{-1},
\end{aligned}
$$

which implies that $z \in \bar{U}\left(x_{-1}, t^{*}-t_{-1}\right)$. Let also $w \in \bar{U}\left(x_{0}, t^{*}-\right.$ $\left.t_{1}\right)$. We get that

$$
\begin{aligned}
\left\|w-x_{0}\right\| & \leq\left\|w-x_{1}\right\|+\left\|x_{1}-x_{0}\right\| \leq t^{*}-t_{1}+t_{1}-t_{0} \\
& =t^{*}-t_{0},
\end{aligned}
$$

hence, $w \in \bar{U}\left(x_{0}, t^{*}, t_{0}\right)$. Note that $\left\|x_{-1}-x_{0}\right\| \leq c=t_{0}-t_{-1}$ and $\left\|x_{1}-x_{0}\right\|=\left\|A_{0}^{-1} F\left(x_{0}\right)\right\| \leq \eta=t_{1}-t_{0}<t^{*}$. That is, $x_{1} \in \bar{U}\left(x_{0}, t^{*}\right) \subseteq \mathscr{D}$. Hence, estimates (67) and (68) hold for $k=-1$ and $k=0$. Suppose that (67) and (68) hold for all $n \leq k$. Then, we obtain that

$$
\begin{aligned}
\left\|x_{k+1}-x_{0}\right\| & \leq \sum_{i=1}^{k+1}\left\|x_{i}-x_{i-1}\right\| \leq \sum_{i=1}^{k+1}\left(t_{i}-t_{i-1}\right) \\
& =t_{k+1}-t_{0}=t^{*}-c \leq t^{*}, \\
\left\|y_{k}-x_{0}\right\| & =\left\|\lambda x_{k}+(1-\lambda) x_{k-1}-x_{0}\right\| \\
& =\left\|\lambda\left(x_{k}-x_{0}\right)+(1-\lambda)\left(x_{k-1}-x_{0}\right)\right\| \\
& \leq \lambda\left\|x_{k}-x_{0}\right\|+(\lambda-1)\left\|x_{k-1}-x_{0}\right\| \\
& \leq \lambda t^{*}+(\lambda-1) t^{*}=(2 \lambda-1) t^{*} .
\end{aligned}
$$

Hence, $x_{k+1}, y_{k} \in \bar{U}\left(x_{0}, t^{*}\right)$.

Using $\left(C_{7}\right)$, Lemma 3 , and the introduction hypotheses, we get that

$$
\begin{aligned}
\| A_{0}^{-1} & \left(A_{k+1}-A_{0}\right) \| \\
\leq & H\left(\left\|y_{k+1}-y_{0}\right\|+\left\|x_{k}-x_{-1}\right\|\right) \\
\leq & H\left(\lambda\left\|x_{k+1}-x_{0}\right\|+|1-\lambda|\left\|x_{k}-x_{-1}\right\|\right. \\
& \left.\quad+\left\|x_{k}-x_{-1}\right\|\right) \\
\leq & H \lambda\left(\left\|x_{k+1}-x_{0}\right\|+\left\|x_{k}-x_{0}\right\|+\left\|x_{0}-x_{-1}\right\|\right) \\
\leq & H \lambda\left(t_{k+1}-t_{0}+t_{k}-t_{0}+c\right) \\
= & H \lambda\left(t_{k+1}+t_{k}-c\right)<1 .
\end{aligned}
$$

It follows from (72) and the Banach lemma on invertible operators $[1,2,4,6-8,14]$ that $A_{k+1}^{-1}$ exists and

$$
\left\|A_{k+1}^{-1} A_{0}\right\| \leq\left(1-H \lambda\left(t_{k+1}+t_{k}-c\right)\right)^{-1} .
$$

In view of (4), we obtain the following identity:

$$
\begin{aligned}
F\left(x_{k+1}\right) & =F\left(x_{k+1}\right)-F\left(x_{k}\right)-\left[y_{k}, x_{k-1} ; F\right]\left(x_{k+1}-x_{k}\right) \\
& =\left(\left[x_{k+1}, x_{k} ; F\right]-\left[y_{k}, x_{k-1} ; F\right]\right)\left(x_{k+1}-x_{k}\right) .
\end{aligned}
$$

Using (4), (34), and the induction hypotheses, we get in turn that

$$
\begin{aligned}
& \left\|A_{0}^{-1} F\left(x_{k+1}\right)\right\| \\
& \quad \leq K\left(\left\|x_{k+1}-y_{k}\right\|+\left\|x_{k}-x_{k-1}\right\|\right)\left\|x_{k+1}-x_{k}\right\| \\
& \quad \leq K\left(\left\|x_{k+1}-x_{k}\right\|+\lambda\left\|x_{k}-x_{k-1}\right\|\right)\left\|x_{k+1}-x_{k}\right\| \\
& \quad \leq K\left(t_{k+1}-t_{k}+\lambda\left(t_{k}-t_{k+1}\right)\right)\left(t_{k+1}-t_{k}\right) .
\end{aligned}
$$

It now follows from (4), (18), (74), and (75) that

$$
\begin{aligned}
\left\|x_{k+2}-x_{k+1}\right\| & \leq\left\|A_{k+1}^{-1} A_{0}\right\|\left\|A_{0}^{-1} F\left(x_{k+1}\right)\right\| \\
& \leq \frac{K\left(t_{k+1}-t_{k}+\lambda\left(t_{k}-t_{k-1}\right)\right)\left(t_{k+1}-t_{k}\right)}{1-H \lambda\left(t_{k+1}+t_{k}-c\right)} \\
& =t_{k+2}-t_{k+1},
\end{aligned}
$$

which completes the induction for (67). Moreover, let $v \in$ $\bar{U}\left(x_{k+2}, t^{*}-t_{k+2}\right)$. Then, we get that

$$
\begin{aligned}
\left\|v-x_{k+1}\right\| & \leq\left\|v-x_{k+2}\right\|+\left\|x_{k+2}-x_{k+1}\right\| \\
& \leq t^{*}-t_{k+2}+t_{k+2}-t_{k+1}=t^{*}-t_{k+1},
\end{aligned}
$$

which implies that $v \in \bar{U}\left(x_{k+1}, t^{*}-t_{k+1}\right)$. The induction for (68) is complete.

Lemma 3 implies that $\left\{t_{k}\right\}$ is a complete sequence. It follows from (67) and (68) that $\left\{x_{k}\right\}$ is a complete sequence in a Banach space $\mathscr{X}$ and as such it converges to some $x^{*} \epsilon$ $\bar{U}\left(x_{0}, t^{*}-c\right)$ (since $\bar{U}\left(x_{0}, t^{*}-c\right)$ is a closed set). By letting $k \rightarrow$ $+\infty$ in (75), we obtain $F\left(x^{*}\right)=0$. Furthermore, estimate (65) follows from (64) by using standard majorization techniques $[1-4,6-8]$. To show the uniqueness part, let $y^{*} \in \bar{U}\left(x_{0}, T\right)$ be such that $F\left(y^{*}\right)=0$. We have that

$$
\begin{aligned}
& \left\|A_{0}^{-1}\left(\left[y^{*}, x^{*} ; F\right]-A_{0}\right)\right\| \\
& \leq H\left(\left\|y^{*}-y_{0}\right\|+\left\|x^{*}-x_{-1}\right\|\right) \\
& \leq H\left(\left\|y^{*}-x_{0}\right\|+(\lambda-1)\left\|x_{0}-x_{-1}\right\|\right. \\
& \left.\quad+\left\|x^{*}-x_{0}\right\|+\left\|x_{0}-x_{-1}\right\|\right) \\
& \leq\left(R_{0}+t^{*}+(\lambda-1) c\right)<1 .
\end{aligned}
$$

It follows from (78) and the Banach lemma on invertible operators that $\left[y^{*}, x^{*} ; F\right]^{-1}$ exists. Then, using the identity $0=F\left(y^{*}\right)-F\left(x^{*}\right)=\left[y^{*}, x^{*} ; F\right]\left(y^{*}-x^{*}\right)$, we deduce that $x^{*}=y^{*}$. The proof of Theorem 8 is complete. 
Remark 9. (a) The limit point $t^{*}$ can be replaced in Theorem 8 by $t^{* *}$ which is given in closed form by (23).

(b) It follows from the proof of Theorem 8 that $\left\{s_{n}\right\}$ is also a majorizing sequence for $\left\{x_{n}\right\}$. Hence, Lemma 4 (or Lemma 7), $\left\{s_{n}\right\}, s^{*}$ can replace Lemma 3 (or Lemma 6) $\left\{t_{n}\right\}, t^{*}$ in Theorem 8 .

Hence, we arrive at the following.

Theorem 10. Suppose that the $(C)$ conditions, Lemma 4 (or Lemma 7), and

$$
U=\bar{U}\left(x_{0},(2 \lambda-1) s^{*}\right) \subseteq \mathscr{D}
$$

hold. Then, sequence $\left\{x_{n}\right\}$ generated by secant-like method is well defined, remains in $U$ for each $n=-1,0,1,2, \ldots$, and converges to a solution $x^{*} \in U\left(x_{0}, s^{*}-c\right)$ of equation $F(x)=0$. Moreover, the following estimates are satisfied for each $n=$ $0,1, \ldots$ :

$$
\begin{gathered}
\left\|x_{n+1}-x_{n}\right\| \leq s_{n+1}-s_{n}, \\
\left\|x_{n}-x^{*}\right\| \leq s^{*}-s_{n} .
\end{gathered}
$$

Furthermore, if there exists $T \geq s^{*}-c$ such that

$$
\begin{gathered}
\bar{U}\left(x_{0}, r\right) \subseteq \mathscr{D}, \\
H\left(T+s^{*}+(\lambda-1) c\right)<1,
\end{gathered}
$$

then, the solution $x^{*}$ is unique in $\bar{U}\left(x_{0}, T\right)$.

Let us consider the following equation:

$$
F(x)+G(x)=0,
$$

where $F$ is a before and $G: \mathscr{D} \rightarrow \mathscr{Y}$ is continuous. The corresponding secant-like method is given by

$x_{n+1}=x_{n}-A_{n}^{-1}\left(F\left(x_{n}\right)+G\left(x_{n}\right)\right)$ for each $n=0,1,2 \ldots$,

where $x_{0}$ is an initial guess.

Suppose that

$$
\left(C_{8}\right)
$$$$
\left\|A_{0}^{-1}(G(x)-G(y))\right\| \leq M\|x-y\| \quad \text { for each } x, y \in \mathscr{D},
$$

$\left(C_{9}\right)$

$$
\left\|A_{0}^{-1}\left(G\left(x_{1}\right)-G\left(x_{0}\right)\right)\right\| \leq M_{0}\left\|x_{1}-x_{0}\right\| .
$$

Clearly,

$$
M_{0} \leq M
$$

holds and $M / M_{0}$ can be arbitrarily large $[1-4,14]$.

We will denote by $\left(C^{*}\right)$ the conditions $(C),\left(C_{8}\right)$, and $\left(C_{9}\right)$. Then, we can present the corresponding result along the same lines as in Lemmas 3, 4, 6, and 7 and Theorems 8 and 10. However, we will only present the results corresponding to Lemma 4 and Theorem 10, respectively. The rest combination of results can be given in an analogous way.
Lemma 11. Let $c \geq 0, \eta>0, H_{0}>0, H_{1}>0, H>0, M_{0}>0$, $M>0, K>0$, and $\lambda \geq 1$. Set $\gamma_{-1}=0, \gamma_{0}=c$, and $\gamma_{1}=c+\eta$. Define scalar sequences $\left\{\gamma_{n}\right\},\left\{\delta_{n}\right\}$ by

$$
\begin{gathered}
\gamma_{2}=\gamma_{1}+\frac{H_{0}\left(\gamma_{1}-\gamma_{0}+\lambda\left(\gamma_{0}-\gamma_{-1}\right)\right)+M_{0}}{1-H_{1} \lambda\left(\gamma_{1}+\gamma_{0}-c\right)}\left(\gamma_{1}-\gamma_{0}\right), \\
\gamma_{n+2}=\gamma_{n+1}+\frac{K\left(\gamma_{n+1}-\gamma_{n}+\lambda\left(\gamma_{n}-\gamma_{n-1}\right)\right)+M}{1-H \lambda\left(\gamma_{n+1}+\gamma_{n}-c\right)}\left(\gamma_{n+1}-\gamma_{n}\right), \\
\delta_{1}=\frac{H_{0}\left(\gamma_{1}-\gamma_{0}+\lambda\left(\gamma_{0}-\gamma_{-1}\right)\right)+M_{0}}{1-H_{1} \lambda\left(\gamma_{1}+\gamma_{0}-c\right)}, \\
\delta_{n}=\frac{K\left(\gamma_{n+1}-\gamma_{n}+\lambda\left(\gamma_{n}-\gamma_{n-1}\right)\right)+M}{1-H \lambda\left(\gamma_{n+1}+\gamma_{n}-c\right)},
\end{gathered}
$$

and functions $h_{n}$ on $[0,1)$ by

$$
\begin{aligned}
h_{n}(t)= & K(t+\lambda) t^{n-1}\left(\gamma_{2}-\gamma_{1}\right)+M \\
& +H \lambda t\left[2 \gamma_{1}+\frac{1-t^{n+1}}{1-t}\left(\gamma_{2}-\gamma_{1}\right)+\frac{1-t^{n}}{1-t}\left(\gamma_{2}-\gamma_{1}\right)\right] \\
& -(1+H \lambda c) t .
\end{aligned}
$$

Suppose that function $\varphi$ given by

$$
\varphi(t)=2 H \lambda\left(\gamma_{1}+\frac{\gamma_{2}-\gamma_{1}}{1-t}\right) t-(1+H \lambda c) t+M
$$

has a minimal zero $a$ in $[0,1)$ and

$$
0 \leq \delta_{1} \leq \alpha \leq a
$$

where $\alpha$ was defined in Lemma 3. Then, sequence $\left\{\gamma_{n}\right\}$ is nondecreasing, bounded from above by $\gamma^{* *}$ that is defined by

$$
\gamma^{* *}=c+\eta+\frac{\gamma_{2}-\gamma_{1}}{1-\alpha}
$$

and converges to its unique least upper bound $\gamma^{*}$ which satisfies

$$
c+\eta \leq \gamma^{*} \leq \gamma^{* *} \text {. }
$$

Moreover, the following estimates are satisfied for each $n=$ $1,2, \ldots$.

$$
0 \leq \gamma_{n+2}-\gamma_{n+1} \leq \alpha^{n}\left(\gamma_{2}-\gamma_{1}\right)
$$

Proof. Simply use $\left\{\gamma_{n}\right\},\left\{\delta_{n}\right\},\left\{h_{n}\right\}, \varphi, a$ instead of $\left\{s_{n}\right\},\left\{b_{n}\right\}$, $\left\{g_{n}\right\}, p, \alpha$ in the proof of Lemma 4 .

Theorem 12. Suppose that the $\left(C^{*}\right)$, Lemma 11 conditions,

$$
U \subseteq \mathscr{D}
$$

hold, where $U$ was defined in Theorem 8 and $\| A_{0}^{-1}\left(F\left(x_{0}\right)+\right.$ $\left.G\left(x_{0}\right)\right) \| \leq \eta$. Then, sequence $\left\{x_{n}\right\}$ generated by the secant-like 
method (83) in well defined, remains in $U$ for each $n=$ $-1,0,1,2, \ldots$, and converges to a solution $x^{*} \in \bar{U}\left(x_{0}, \gamma^{*}-c\right)$ of equation $F(x)+G(x)=0$. Moreover, the following estimates are satisfied for each $n=0,1, \ldots$ :

$$
\begin{gathered}
\left\|x_{n+1}-x_{n}\right\| \leq \gamma_{n+1}-\gamma_{n}, \\
\left\|x_{n}-x^{*}\right\| \leq \gamma^{*}-\gamma_{n} .
\end{gathered}
$$

Furthermore, if there exists $\bar{\gamma} \geq \gamma^{*}-c$ such that

$$
\begin{gathered}
\bar{U}\left(x_{0}, \bar{\gamma}\right) \subseteq \mathscr{D}, \\
0<\frac{K((\lambda-1) c+\bar{\gamma})+M}{1-H \lambda(2 \bar{\gamma}-c)} \leq \eta, \quad \text { for some } \mu \in(0,1),
\end{gathered}
$$

then, the solution $x^{*}$ is unique in $\bar{U}\left(x_{0}, \bar{\gamma}\right)$

Proof. The proof until the uniqueness part follows as in Theorem 8 but using the following identity:

$$
\begin{aligned}
& F\left(x_{k+1}\right)+G\left(x_{k+1}\right) \\
& \quad=\left(\left[x_{k+1}, x_{k} ; F\right]-A_{k}\right)\left(x_{k+1}-x_{k}\right)+\left(G\left(x_{k+1}\right)-G\left(x_{k}\right)\right)
\end{aligned}
$$

instead of (74). Finally, for the uniqueness part, let $y^{*} \epsilon$ $\bar{U}\left(x_{0}, \bar{\gamma}\right)$ be such that $F\left(y^{*}\right)+G\left(y^{*}\right)=0$. Then, we get from (83) the identity

$$
\begin{aligned}
x_{n+1}-y^{*}=x_{n}-A_{n}^{-1}\left(F\left(x_{n}\right)+\left(x_{n}\right)\right)-y^{*} \\
=-A_{n}^{-1}\left(F\left(x_{n}\right)-F\left(x^{*}\right)-A_{n}\left(x_{n}-y^{*}\right)\right. \\
\left.\quad+\left(G\left(x_{n}\right)-G\left(y^{*}\right)\right)\right) \\
=-A_{n}^{-1}\left(\left(\left[x_{n}, y^{*} ; F\right]-\left[y_{n}, x_{n-1} ; F\right]\right)\left(x_{n}-y^{*}\right)\right. \\
\left.+G\left(x_{n}\right)-G\left(y^{*}\right)\right) .
\end{aligned}
$$

This identity leads to

$$
\begin{aligned}
\left\|x_{n+1}-y^{*}\right\| \leq & \frac{K\left(\left\|x_{n}-y_{n}\right\|+\left\|x_{n-1}-y^{*}\right\|\right)+M}{1-H \lambda\left(\gamma_{n+1}+\gamma_{n}-c\right)}\left\|x_{n}-y^{*}\right\| \\
\leq & \frac{K\left((\lambda-1)\left\|x_{n}-x_{n-1}\right\|+\left\|x_{n-1}-y^{*}\right\|\right)+M}{1-H \lambda(2 \bar{\gamma}-c)} \\
& \times\left\|x_{n}-y^{*}\right\| \\
\leq & \frac{K((\lambda-1) c+\bar{\gamma})+M}{1-H \lambda(2 \bar{\gamma}-c)}\left\|x_{n}-y^{*}\right\| \\
\leq & \mu\left\|x_{n}+y^{*}\right\| \\
\leq & \mu^{n+1}\left\|x_{0}-y^{*}\right\| \leq \mu^{n+1} \bar{\gamma} .
\end{aligned}
$$

Hence, we deduce $\lim _{n \rightarrow+\infty} x_{n}=y^{*}$. But we know that $\lim _{n \rightarrow+\infty} x_{n}=x^{*}$. That is, we conclude that $x^{*}=y^{*}$. That completes the proof of the theorem.

\section{Numerical Examples}

Example 1. Let $\mathscr{X}=\mathscr{y}=\mathscr{C}[0,1]$, equipped with the max-norm. Consider the following nonlinear boundary value problem:

$$
\begin{gathered}
u^{\prime \prime}=-u^{3}-\gamma u^{2}, \\
u(0)=0, \quad u(1)=1 .
\end{gathered}
$$

It is well known that this problem can be formulated as the integral equation

$$
u(s)=s+\int_{0}^{1} Q(s, t)\left(u^{3}(t)+\gamma u^{2}(t)\right) d t
$$

where $Q$ is the Green function as follows:

$$
Q(s, t)= \begin{cases}t(1-s), & t \leq s \\ s(1-t), & s<t\end{cases}
$$

We observe that

$$
\max _{0 \leq s \leq 1} \int_{0}^{1}|\mathcal{Q}(s, t)| d t=\frac{1}{8} .
$$

Then, problem (101) is in the form (1), where $F: \mathscr{D} \rightarrow \mathscr{Y}$ is defined as

$$
[F(x)](s)=x(s)-s-\int_{0}^{1} \mathscr{Q}(s, t)\left(x^{3}(t)+\gamma x^{2}(t)\right) d t .
$$

The Fréchet derivative of the operator $F$ is given by

$$
\begin{gathered}
{\left[F^{\prime}(x) y\right](s)=y(s)-3 \int_{0}^{1} \mathscr{Q}(s, t) x^{2}(t) y(t) d t} \\
-2 \gamma \int_{0}^{1} \mathbb{Q}(s, t) x(t) y(t) d t .
\end{gathered}
$$

Then, we have that

$$
\begin{aligned}
{\left[\left(I-F^{\prime}\left(x_{0}\right)\right)(y)\right](s)=} & 3 \int_{0}^{1} \mathscr{Q}(s, t) x_{0}^{2}(t) y(t) d t \\
& +2 \gamma \int_{0}^{1} \widehat{Q}(s, t) x_{0}(t) y(t) d t
\end{aligned}
$$

Hence, if $2 \gamma<5$, then

$$
\left\|I-F^{\prime}\left(x_{0}\right)\right\| \leq 2(\gamma-2)<1 .
$$

It follows that $F^{\prime}\left(x_{0}\right)^{-1}$ exists and

$$
\left\|F^{\prime}\left(x_{0}\right)^{-1}\right\| \leq \frac{1}{5-2 \gamma} .
$$

We also have that $\left\|F\left(x_{0}\right)\right\| \leq 1+\gamma$. Define the divided difference defined by

$$
\delta F(x, y)=\int_{0}^{1} F^{\prime}(y+t(x-y)) d t .
$$


Choosing $x_{-1}(s)$ such that $\left\|x_{-1}-x_{0}\right\| \leq c$ and $l_{0} c<1$. Then, we have for $\lambda=1$,

$$
\begin{aligned}
\left\|\delta F\left(x_{-1}, x_{0}\right)^{-1} F\left(x_{0}\right)\right\| \leq & \left\|\delta F\left(x_{-1}, x_{0}\right)^{-1} F^{\prime}\left(x_{0}\right)\right\| \\
& \times\left\|F^{\prime}\left(x_{0}\right) F\left(x_{0}\right)\right\|, \\
\left\|\delta F\left(x_{-1}, x_{0}\right)^{-1} F^{\prime}\left(x_{0}\right)\right\| & \leq \frac{1}{\left(1-l_{0} c\right)},
\end{aligned}
$$

where $l_{0}$ is such that

$$
\left\|F^{\prime}\left(x_{0}\right)^{-1}\left(F^{\prime}\left(x_{0}\right)-A_{0}\right)\right\| \leq l_{0} c .
$$

Set $u_{0}(s)=s$ and $\mathscr{D}=U\left(u_{0}, R_{0}\right)$. It is easy to verify that $U\left(u_{0}, R_{0}\right) \subset U\left(0, R_{0}+1\right)$ since $\left\|u_{0}\right\|=1$. If $2 \gamma<5$ and $l_{0} c<1$, the operator $F^{\prime}$ satisfies conditions of Theorem 8, with

$$
\begin{gathered}
\eta=\frac{1+\gamma}{\left(1-l_{0} c\right)(5-2 \gamma)}, \quad K=\frac{\gamma+6 R_{0}+3}{8(5-2 \gamma)\left(1-l_{0} c\right)}, \\
H=\frac{2 \gamma+3 R_{0}+6}{16(5-2 \gamma)\left(1-l_{0} c\right)} .
\end{gathered}
$$

Choosing $R_{0}=1, \gamma=0.5$, and $c=1$, we obtain that

$$
\begin{gathered}
l_{0}=0.1938137822 \ldots, \\
\eta=0.465153 \ldots, \\
K=0.368246 \ldots, \\
H=0.193814 \ldots
\end{gathered}
$$

Moreover, we obtain that $a_{-1}=0.317477$ and $b_{-1}=$ 0.251336 , but conditions of Theorem 1 are not satisfied since

$$
b_{-1}=0.251336>0.147893=\frac{a_{-1}\left(1-a_{-1}\right)^{2}}{2\left(1-a_{-1}\right)-\lambda\left(1-2 a_{-1}\right)} .
$$

Notice also that the popular condition (53) is also not satisfied, since $K c+2 \sqrt{K \eta}=1.19599>1$. Hence, there is no guarantee under the old conditions that the secant-type method converges to $x^{*}$. However, conditions of Lemma 3 are satisfied since

$$
0<\alpha=0.724067 \leq 0.776347=\frac{1-H \lambda(c+2 \eta)}{1-H \lambda c} .
$$

The convergence of the secant-type method is also ensured by Theorem 8.

Example 2. Let $\mathscr{X}=\mathscr{Y}=\mathbb{R}$, and consider the real function

$$
F(x)=x^{3}-2,
$$

and we are going to apply secant-type method with $\lambda=2.5$. We take the starting points $x_{0}=1, x_{-1}=0.25$ and we consider the domain $\Omega=B\left(x_{0}, 3 / 4\right)$. In this case, we obtain

$$
\begin{gathered}
c=0.75, \\
\eta=0.120301 \ldots, \\
K=0.442105 \ldots, \\
H=0.180451 \ldots .
\end{gathered}
$$

Notice that the conditions of Theorem 1 and Lemma 3 are satisfied, but since $H<K$, Remark 2 ensures that our uniqueness ball is larger. It is clear as $R_{1}=1.83333 \cdots>$ $0.193452 \cdots=R_{0}$.

\section{Conflict of Interests}

The authors declare that there is no conflict of interests regarding the publication of this paper.

\section{References}

[1] I. K. Argyros, Convergence and Applications of Newton-Type Iterations, Springer, New York, NY, USA, 2008.

[2] I. K. Argyros, Y. J. Cho, and S. Hilout, Numerical Methods for Equations and Its Applications, CRC Press/Taylor \& Francis, Boca Raton, Fla, USA, 2012.

[3] I. K. Argyros and S. Hilout, "Weaker conditions for the convergence of Newton's method," Journal of Complexity, vol. 28, no. 3, pp. 364-387, 2012.

[4] I. K. Argyros, S. Hilout, and M. A. Tabatabai, Mathematical Modeling with Applications in Biosciences and Engineering, Nova, New York, NY, USA, 2011.

[5] J. A. Ezquerro, M. Grau-Sánchez, M. A. Hernández, and M. Noguera, "Semilocal convergence of secant-like methods for differentiable and nondifferentiable operator equations," Journal of Mathematical Analysis and Applications, vol. 398, no. 1, pp. 100-112, 2013.

[6] F. A. Potra and V. Pták, Nondiscrete Induction and Iterative Processes, vol. 103 of Research Notes in Mathematics, Pitman, Boston, Mass, USA, 1984.

[7] J. F. Traub, Iterative Methods for the Solution of Equations, Prentice-Hall, Englewood Cliffs, NJ, USA, 1964.

[8] P. Laasonen, "Ein überquadratisch konvergenter iterativer algorithmu," Annales Academiae Scientiarum Fennicae Mathematica I, vol. 450, p. 110, 1969.

[9] J. M. Ortega and W. C. Rheinboldt, Iterative Solution of Nonlinear Equations in Several Variables, Academic Press, New York, NY, USA, 1970.

[10] J. W. Schmidt, "Untere fehlerschranken für regula-falsi-verfahren," Periodica Mathematica Hungarica, vol. 9, no. 3, pp. 241247, 1978.

[11] S. Amat, C. Bermúdez, S. Busquier, and J. O. Gretay, "Convergence by nondiscrete mathematical induction of a two step secant's method," Rocky Mountain Journal of Mathematics, vol. 37, no. 2, pp. 359-369, 2007.

[12] S. Amat, S. Busquier, and J. M. Gutiérrez, "On the local convergence of secant-type methods," International Journal of Computer Mathematics, vol. 81, no. 9, pp. 1153-1161, 2004.

[13] S. Amat, S. Busquier, and M. Negra, "Adaptive approximation of nonlinear operators," Numerical Functional Analysis and Optimization, vol. 25, no. 5-6, pp. 397-405, 2004.

[14] I. K. Argyros, Polynomial Operator Equations in Abstract Spaces and Applications, Mathematics Series, St. Lucie/CRC Press/Lewis, Boca Raton, Fla, USA, 1998.

[15] I. K. Argyros and S. Hilout, "Convergence conditions for secanttype methods," Czechoslovak Mathematical Journal, vol. 60, no. 1, pp. 253-272, 2010.

[16] W. E. Bosarge, Jr. and P. L. Falb, "A multipoint method of third order," Journal of Optimization Theory and Applications, vol. 4, pp. 156-166, 1969. 
[17] J. E. Dennis, "Toward a unified convergence theory for Newtonlike methods," in Functional Analysis and Applications, L. B. Rall, Ed., Academic Press, New York, NY, USA, 1971.

[18] J. A. Ezquerro, J. M. Gutiérrez, M. A. Hernández, N. Romero, and M. J. Rubio, "The Newton method: from Newton to Kantorovich," La Gaceta de la Real Sociedad Matemática Española, vol. 13, no. 1, pp. 53-76, 2010 (Spanish).

[19] J. A. Ezquerro, M. A. Hernández, N. Romero, and A. I. Velasco, "Improving the domain of starting points for secantlike methods", Applied Mathematics and Computation, vol. 219, no. 8, pp. 3677-3692, 2012.

[20] M. A. Hernández, M. J. Rubio, and J. A. Ezquerro, "Secant-like methods for solving nonlinear integral equations of the Hammerstein type," Journal of Computational and Applied Mathematics, vol. 115, pp. 245-254, 2000.

[21] M. A. Hernández, M. J. Rubio, and J. A. Ezquerro, "Solving a special case of conservative problems by secant-like methods," Applied Mathematics and Computation, vol. 169, no. 2, pp. 926942, 2005.

[22] M. A. Hernández and M. J. Rubio, "A uniparametric family of iterative processes for solving nondifferentiable equations," Journal of Mathematical Analysis and Applications, vol. 275, no. 2, pp. 821-834, 2002.

[23] L. V. Kantorovich and G. P. Akilov, Functional Analysis, Pergamon Press, Oxford, UK, 2nd edition, 1982.

[24] P. D. Proinov, "General local convergence theory for a class of iterative processes and its applications to Newton's method," Journal of Complexity, vol. 25, no. 1, pp. 38-62, 2009.

[25] T. Yamamoto, "A convergence theorem for Newton-like methods in Banach spaces," Numerische Mathematik, vol. 51, no. 5, pp. 545-557, 1987.

[26] M. A. Wolfe, "Extended iterative methods for the solution of operator equations," Numerische Mathematik, vol. 31, no. 2, pp. 153-174, 1979. 


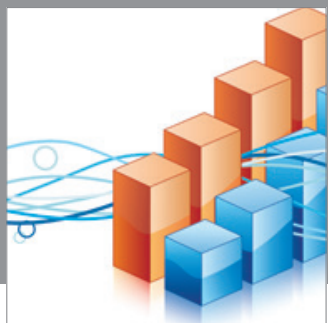

Advances in

Operations Research

mansans

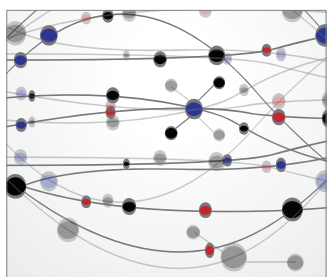

The Scientific World Journal
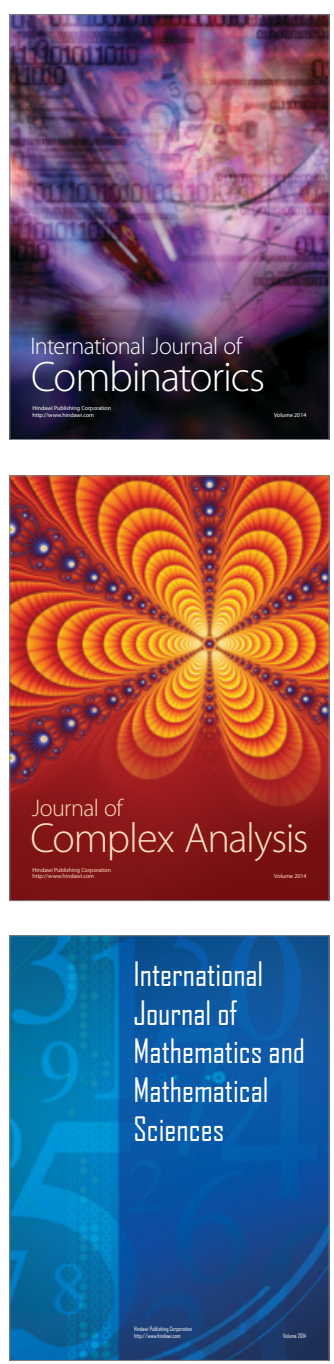
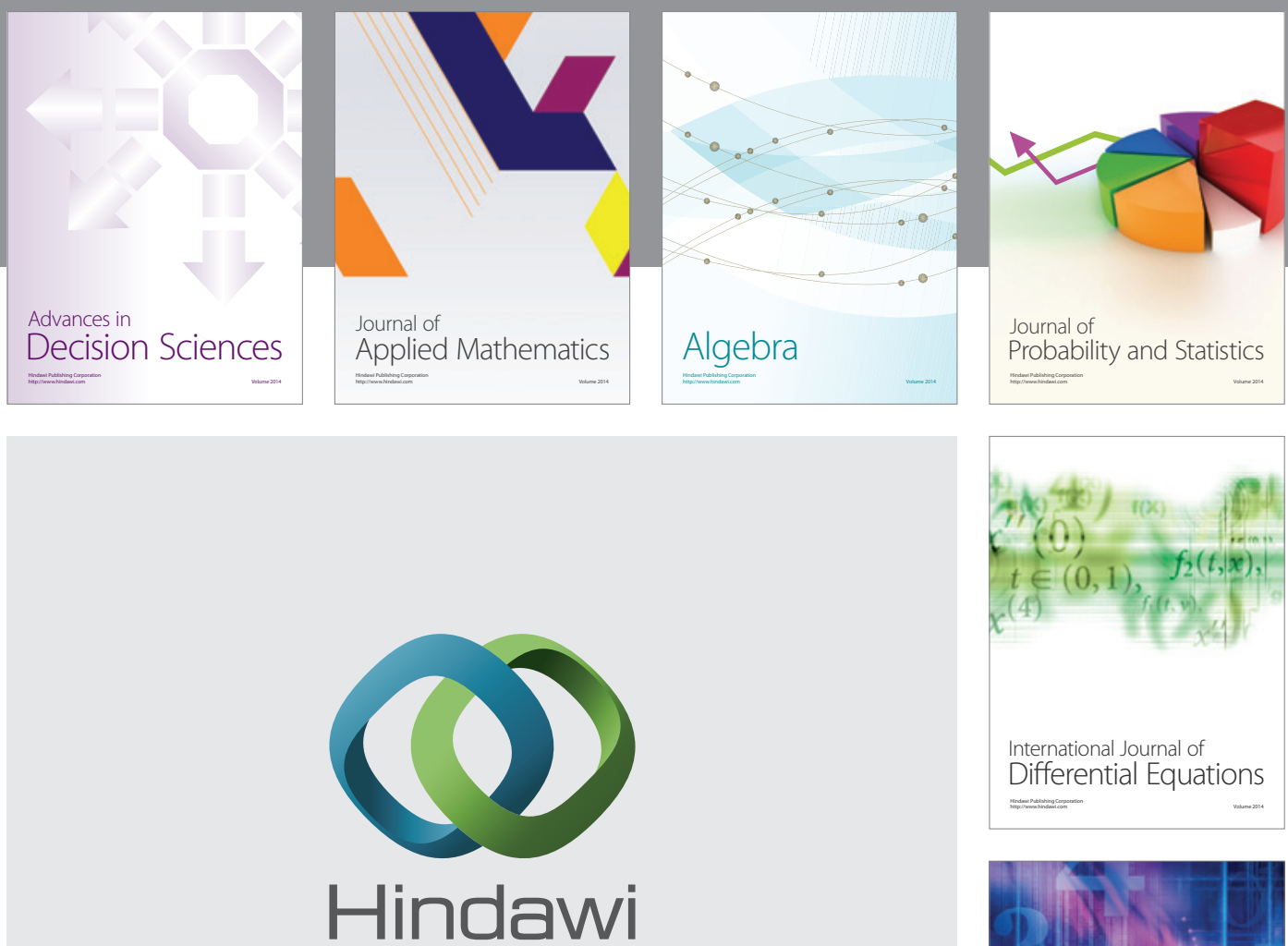

Submit your manuscripts at http://www.hindawi.com
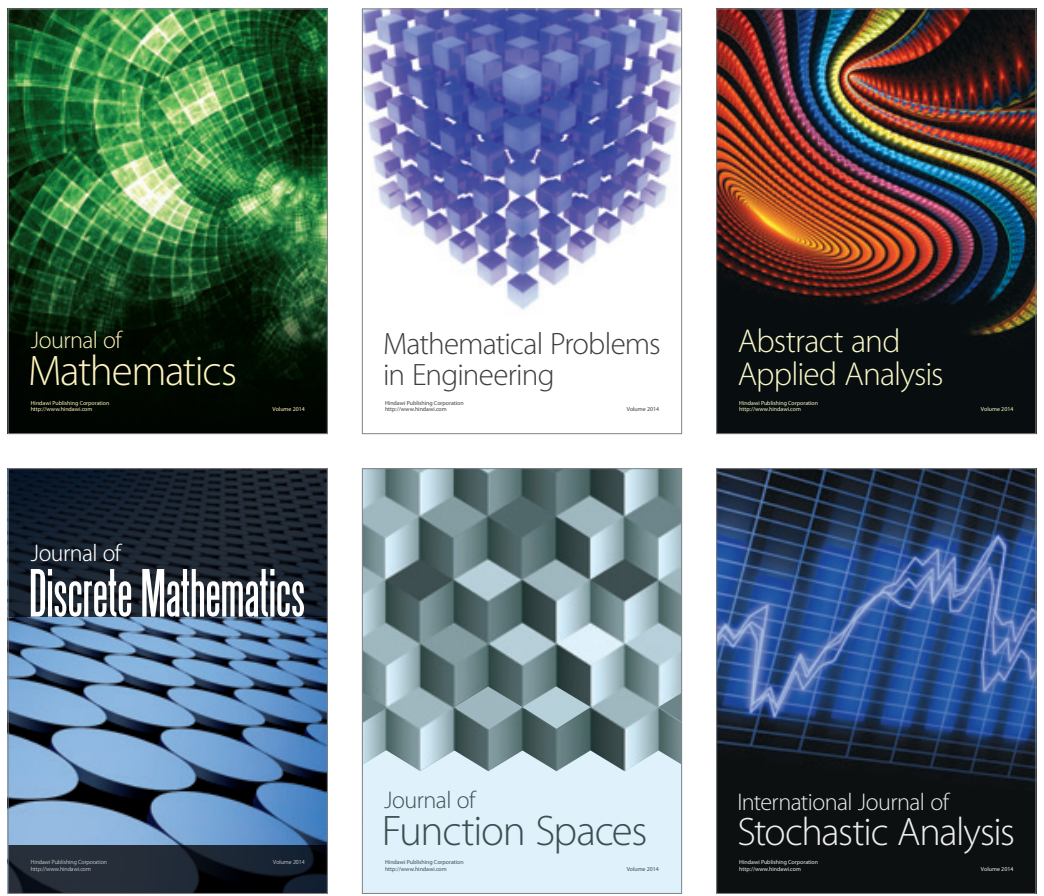

Journal of

Function Spaces

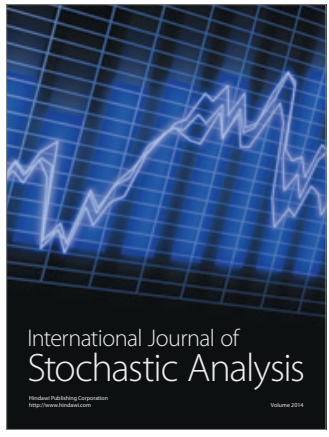

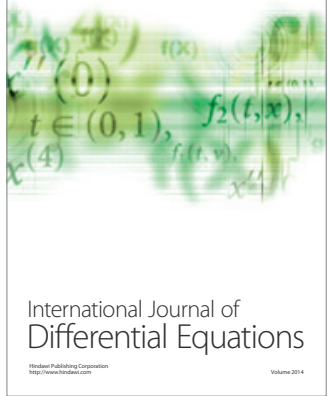
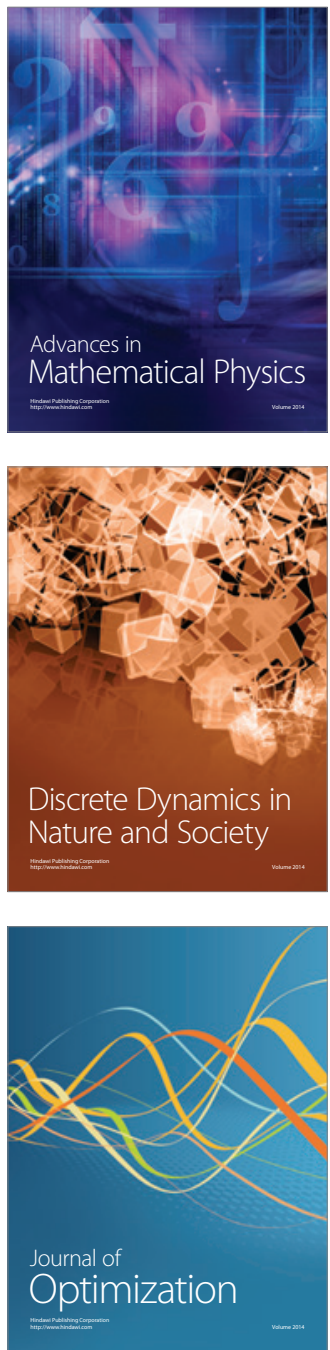